\title{
Update on the Neuroimaging and Electroencephalographic Biomarkers of Epileptogenesis: A Literature Review
}

\author{
Guihua Chen ${ }^{1+}$, Zheyu Zhang ${ }^{2 t}$, Meiping Wang ${ }^{1}$, Yu Geng ${ }^{1}$, Bo Jin ${ }^{1 *}$ and Thandar Aung ${ }^{3}$ \\ 'Department of Neurology, Zhejiang Provincial People's Hospital, People's Hospital of Hangzhou Medical College, \\ Hangzhou, China, ${ }^{2}$ The Second Clinical Medical College, Zhejiang Chinese Medical University, Hangzhou, China, ${ }^{3}$ Epilepsy \\ Center, University of Pittsburgh, Pittsburgh, PA, United States
}

\section{OPEN ACCESS}

Edited by:

Pasquale Striano, University of Genoa, Italy

Reviewed by:

Antonella Riva,

University of Genoa, Italy Antonio Leo,

Università Magna Grecia di

Catanzaro, Italy

*Correspondence:

Bo Jin

jinbo0111@outlook.com

†These authors have contributed equally to this work

Specialty section: This article was submitted to

Epilepsy,

a section of the journal

Frontiers in Neurology

Received: 09 July 2021 Accepted: 09 August 2021

Published: 27 August 2021

Citation:

Chen G, Zhang Z, Wang M, Geng Y, Jin B and Aung T (2021) Update on the Neuroimaging and Electroencephalographic Biomarkers of Epileptogenesis: A Literature Review. Front. Neurol. 12:738658. doi: 10.3389/fneur.2021.738658
Epilepsy is one of the most common debilitating neurological disorders that lead to severe socio-cognitive dysfunction. While there are currently more than 30 antiseizure medications available for the treatment and prevention of seizures, none address the prevention of epileptogenesis that leading to the development of epilepsy following a potential brain insult. Hence, there is a growing need for the identification of accurate biomarkers of epileptogenesis that enable the prediction of epilepsy following a known brain insult. Although recent studies using various neuroimages and electroencephalography have found promising biomarkers of epileptogenesis, their utility needs to be further validated in larger clinical trials. In this literature review, we searched the Medline, Pubmed, and Embase databases using the following search algorithm: "epileptogenesis" and "biomarker" and "EEG" or "electroencephalography" or "neuroimaging" limited to publications in English. We presented a comprehensive overview of recent innovations in the role of neuroimaging and EEG in identifying reliable biomarkers of epileptogenesis.

Keywords: epilepsy, epileptogenesis, biomarker, neuroimage, EEG

\section{INTRODUCTION}

Epilepsy is one of the most common neurological disorders affecting around 70 million people worldwide. Approximately 2.4 million new cases of epilepsy are diagnosed annually (1). Subsequently, epilepsy is getting increased public health attention as patients with epilepsy have a noticeable reduction in quality of life and employment prospects (2). Although antiseizure medication (ASMs) are considered the first-line treatment of epilepsy, it is still widely recognized that nearly one-third of epileptic patients have drug-resistant epilepsy in which seizures are unable to be controlled with at least two appropriately ASMs (3). One potential reason is that current accessible ASMs merely prevent one from having further spontaneous seizures but do not directly affect or alter the underlying cause contributing to epileptogenesis $(4,5)$. Through plentiful scientific research on the pathophysiology of epilepsy over the past several decades, there has been an increasing understanding of the pathophysiology of epileptogenesis.

Epileptogenesis refers to the process by which normal brain tissue becomes capable of generating spontaneous recurrent seizures and its progression (6). With the advances in technology, potential promising biomarkers are now available to predict the development of epileptogenesis after 
the epileptogenic insult. Furthermore, there are accessible therapeutic biomarkers that predict the treatment prognosis through the identification and exact localization of the epileptogenic lesion and associated network, as well as the severity and progression of epileptogenesis $(7,8)$. The ideal biomarkers should not just have validity, reliability, and reproducibility; they should also be non-invasive and costeffective (9). Among all the available biomarkers related to epileptogenesis, neuroimaging and electroencephalogram (EEG) biomarkers are by far the most appealing biomarkers as they are non-invasive and routinely performed as part of epileptic patients' workup protocol.

In this review article, we searched the Medline, Pubmed, and Embase databases using the following search algorithm: "epileptogenesis" and "biomarker" and "EEG" or "electroencephalography" or "neuroimaging" limited to publications in English. The last date of the search was May 31, 2021. We screened the titles, abstracts, and references of all search results to identify potentially relevant studies. We aimed to provide a comprehensive literature review of recent innovations in the role of neuroimaging and EEG as biomarkers of the development of epileptogenesis after the epileptogenic insult.

\section{NEUROIMAGING BIOMARKERS}

Several neuroimaging modalities, including structural magnetic resonance imaging (MRI), functional MRI, magnetic resonance spectroscopy (MRS), and positron emission tomography (PET), have already been applied to investigate biomarkers of epileptogenesis and have significantly contributed to our understanding of the pathophysiological mechanisms that underlie the development of epilepsy (Table 1).

\section{Structural MRI}

MRI is an ideal tool for biomarker studies due to its accessibility and translatability to routine clinic settings. In the lateral fluid percussion injury (LFPI) rat model of traumatic brain injury (TBI), the abnormalities in the surface morphology of the ipsilateral hippocampus at 1-week post-LFPI can predict the occurrence of epilepsy 6 months after TBI (10). Besides, assessing individual MRI parameters in the peri-lesional cortex or the thalamus at 9 days after TBI can also provide high sensitivity and specificity for predicting increased seizure susceptibility at 12 months (11). In the follow-up LFPI study, the presence of diffusion abnormality analyzed by using $\mathrm{D}_{\mathrm{av}}$ in the perilesional cortex and thalamus at 2 months after the TBI is found to have the highest predictive value for the development of seizure susceptibility at 12 months post-TBI. Similar changes in the MRI structures have been validated at the human level. In the early acute post-TBI phase (within 90 days postinjury), there is evidence showing a positive correlation between hippocampal/temporal structural abnormalities and the onset of seizure activity (11). In addition to the injury severity, the left temporal pole and left frontal cortical thinness are found to be significantly predictive factors for developing seizures after TBI (12). In addition to the cortical and subcortical structures, studies have shown that the distribution and quantification of paravscular spaces (PVSs) can be used as a potential biomarker for the development of epileptogenesis in posttraumatic epilepsy (PTE). Post-TBI epileptic patients are found to have significantly smaller PVSs and asymmetric distribution of PVSs in the suspected epileptogenic hemisphere $(13,14)$.

Mesial temporal lobe epilepsy (TLE) is reported as a common sequelae of the febrile status epilepticus (FSE) (38). Recently, in the rat model of FSE, reduced $\mathrm{T} 2$ relaxation time in the amygdala within $2 \mathrm{~h}$ of FSE is observed in the high resolution 11.7T MRI. This finding is shown to have a strong prediction of the later occurrence of TLE following the FSE. It is hypothesized that T2 changes are related to the increased oxygen utilization after FSE termination, which correlates with the activation of the intracellular inflammatory cascades that had been previously implicated in epileptogenesis (15). The result is again validated in the lower resolution 3T MRI (16). These results suggest that the quantitative T2 MRI can be used as a reliable neuroimaging biomarker following FSE for brain injury and structural alterations at the onset of epileptogenesis. Further studies are warranted to validate the reduction of the T2 relaxation time in the amygdala in the development of epileptogenesis in humans before they are ready for the clinical setting. Overall the translational nature of the MRI results has also greatly contributed to clinical advancements as the reported neuro-imaging protocols can be applied safely to epileptic patients.

Diffusion-weighted imaging (DWI) represents the diffusion of water molecules and is particularly useful for detecting acute changes in the brain tissue following status epilepticus (SE) (17). Yokoi and colleagues analyzed the acute DWI data of 22 children with FSE over the period of 9-13 years following FSE and reported that focal epilepsy was significantly more frequent in patients with hippocampal DWI hyperintensity than those without DWI changes (39). Further studies, including a larger cohort, are required to investigate the relationship between DWI changes and subsequent epilepsy in patients with FSE.

Diffusion tensor imaging (DTI) detects the restriction of water diffusion caused by the microstructural organization of tissues. Advancement in post-processing technique allows DTI to detect subtle white matter changes in the early development of epileptogenesis in a structural network-based approach (40). Sierra and colleagues studied and compared the fractional anisotropy (FA) and axial, radial, and mean diffusivities among three subgroups of rats: SE, TBI, and normal controls, at 612 months post-injury using 9.4T MRI. FA in the hippocampi was significantly increased in the SE/TBI group compared to the normal control group. Regarding the diffusivities, there was an increase in the $D_{\|}$(associated with axonal damage) after the $\mathrm{SE}$, whereas a decrease in $D_{\perp}$ (associated with demyelination) was noted after the TBI in the subfield-specific hippocampi. Thus, the data suggest that the DTI method identifies not only subtle hippocampal changes and progression after epileptogenic brain injuries but also different brain insults based on the different diffusivities (18). Several recent longitudinal studies of the mesial TLE rate models demonstrated that the epileptogenic rats have significant changes in DTI-measured FA in the early 
TABLE 1 | Overview of neuroimaging biomarkers of epileptogenesis.

\begin{tabular}{|c|c|c|c|}
\hline Imaging modality & Animal model & Human epilepsy & References \\
\hline \multicolumn{4}{|l|}{ Structural MRI } \\
\hline Surface morphology & LFPI model & None & (10) \\
\hline $\mathrm{D}_{\mathrm{av}}$ & LFPI model & None & (11) \\
\hline Cortical thinness & None & PTE & (12) \\
\hline PVSs & None & PTE & $(13,14)$ \\
\hline \multirow[t]{3}{*}{ DTI } & LFPI model & None & (18) \\
\hline & Pilocarpine -induced SE mode & None & (18) \\
\hline & MSO infused model & - & (19) \\
\hline Functional MRI & & & (20) \\
\hline Global network & KA-induced SE model & None & $(21-23)$ \\
\hline GABA-A & Pilocarpine -induced SE mode & None & (26) \\
\hline Myo-inositol & Pilocarpine -induced SE mode & None & $(25,27)$ \\
\hline Antioxidant glutathione & Pilocarpine -induced SE mode & None & (25) \\
\hline \multicolumn{4}{|l|}{ PET } \\
\hline \multirow[t]{3}{*}{ 18F-FDG } & LFPI model & None & (10) \\
\hline & KA-induced SE model & None & (23-32) \\
\hline & Pilocarpine-induced SE model & None & (30) \\
\hline GABA-A & KA-induced SE model & None & $(33-35)$ \\
\hline TSPO & KA-induced SE model & None & $(36,37)$ \\
\hline
\end{tabular}

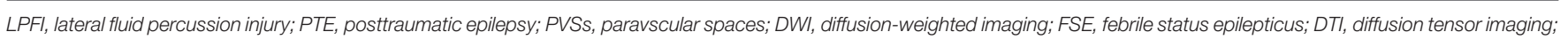
KA, kainic acid; SE, status epilepticus; MSO, methionine sulfoximine; NAA, N-acetyl aspartate; TSPO, 18-kDa translocator protein.

stages. In addition, the FA changes in both gray and white matter progress over time as the animals transitioned from early to late epileptogenesis (19). Although the results suggest that DTI changes can be used as biomarkers of epileptogenesis, no prospective studies of epileptogenesis with DTI have been implemented in humans. Thus, further studies are warranted to implement the finding in the clinical setting.

\section{Functional MRI}

Functional MRI detects hemodynamic changes in different parts of the brain by means of blood oxygen level-dependent (BOLD) sequences, an indirect non-invasive measure of neuronal activity (41). Contrary to measuring the structural connectivity with DTI, functional MRI measures functional connectivity between various brain regions. Using the animal intrahippocampal kainic acid (KA) model of mesial TLE, Li et al. compared the fMRI of animals with mesial TLE and animals without epilepsy at 1 week after SE. For global network features, animals with epilepsy showed an overall increase in functional connectivity strength compared to animals without epilepsy. For local network features, animals without epilepsy showed decreased hubness in the hippocampus, whereas animals with epilepsy showed a complete loss of hippocampus hubs with appearance of new hubs in the prefrontal cortex (21). Instead of the hypersynchrony brain network pattern, Christiaen et al. reported a decreased functional connectivity between 1 and 3 weeks post SE after comparing 20 intraperitoneal KA animals and seven healthy control animals (22). The difference might be the result of the different routes of administration of ketamine as intraperitoneal injection resulted in more widespread brain lesions than intrahippocampal injection (21). Furthermore, Bertoglio et al. demonstrated diverging changes in network connectivity in relation to the seizure onset in the KA-induced models of SE. Animals with regular seizure onset ( $<17$ days postSE) showed a significant hypersynchrony of network connectivity at 4 weeks post-SE, while animals with delayed disease onset $(\geq 17$ days post-SE) remained hyposynchronous (23). Although there is a discrepancy across different studies on functional connectivity after TBI or TLE, the current literature suggests that there may be a reorganization of the functional network in early period of epileptogenesis, which may be used as an imaging biomarker in the near future.

\section{MRS}

MRS can provide indirect information, such as neuronal health, gliosis, energy metabolism, by analyzing different metabolites in the brain tissue. Several studies have analyzed the changes of $\mathrm{N}$ -acetyl aspartate (NAA) and gamma-aminobutyric acid (GABA) in the pilocarpine-induced SE model, a reduced NAA and GABA can be detected in the hippocampus from baseline to the period of epileptic seizures (24-26). However, a decrease in GABA and NAA levels has also been found in patients after TBI without 
correlation with epileptic seizures (28). A progressive increase in myo-inositol and antioxidant glutathione before the onset of seizures has been found in pilocarpine-induced SE $(25,27)$. Furthermore, the level of antioxidant glutathione has shown to be negatively correlated with the frequency of spontaneous seizures (25). Although MRS analyses in seizure-prone brain areas following potential epileptogenic injuries may represent clinically meaningful biomarkers for the early identification of individuals at high risk for developing epilepsy, further human studies are warranted to validate the finding for the clinical setting.

\section{PET}

Alteration in the brain metabolic activity has been reported in the early development of the epileptogenesis after the initial epileptic insults. Nuclear imaging modalities such as PET are optimally used to assess functional brain metabolic activity using various radiotracers. Hence, PET has been used in several studies to assess potential mechanisms of epileptogenesis.

In the LFPI model, epileptic rats' ipsilateral hippocampi are reported to have subtle thickening on the surface analysis and 18F-FDG PET hypometabolism at 1 week, 1 month, and 3 months post-injury compared to the non-epileptic group. In addition, all the TBI rats have reported cortical and hippocampal hypometabolism, but the non-epileptic group has a partial recovery of the FDG uptake at 3 months post-injury (10). Thus, an initial reduction in glucose uptake is perhaps the result of both injury itself and early epileptogenesis, but in epileptic rats, there would be no recovery of the initial hypometabolism (29).

Multiple studies using KA and pilocarpine-induced models of SE have shown an initial increase in the glucose uptake during the acute seizures followed by the reduced metabolism at around day 3 of post-SE $(24,30)$. Furthermore, in the KA models of SE, glucose hypometabolism during early epilepsy correlates with the duration of the latent phase and frequency of spontaneous seizures in the spontaneous recurrent seizure (SRS) model of epilepsy $(31,32)$.

Several studies used different PET radioligands other than 18 F-FDG to investigate the relationship between the density of GABA-A receptors and epileptogenesis in animal models of epilepsy. It is widely observed that GABA-A receptor density is decreased not only in hippocampi but also in several cortical regions in the KA models of $\operatorname{SE}(33,34)$. One study using the focal cortical dysplasia model suggests that the decrease in the GABA-A receptor density may characterize a latent phase of epileptogenesis (35). Further studies are warranted to get more validation, but the promising results suggest that glucose hypometabolism and reduced GABA-A receptors might be the important hallmarks of early epileptogenesis.

Neuroinflammation is another pathological hallmark in one of the major pathophysiologies for epileptogenesis (42). The investigation of inflammation can be performed using a PET scan with radioligands that bind to $18-\mathrm{kDa}$ translocator protein (TSPO). TSPO is reported to be highly expressed on the mitochondrial membrane of activated microglia and reactive astrocytes. In the SRS model of epilepsy, TSPO levels at 14 days post-SE are predictive of SRS frequency at the onset of epilepsy (36). Subsequently, the same researcher group reported that TSPO upregulation at 14 days post-SE was associated with epileptogenesis, while TSPO overexpression at 14 days post-SE was associated with seizure frequency (37). Although TSPO-PET results are promising, clinical PET data on this topic is very limited due to the cost and availability of specific radioligands.

\section{EEG}

EEG, either non-invasive scalp recording or invasive microelectrode recording, is one of the most utilized modalities in the clinical setting and can monitor brain activity with high temporal resolution and relatively high spatial resolution. Several studies have demonstrated specific changes in the EEG as the potential biomarkers for the early development of epileptogenesis (Table 2).

\section{High-Frequency Oscillations (HFOs)}

HFOs, i.e., ripples $(80-250 \mathrm{~Hz})$ and fast ripples $(250-600 \mathrm{~Hz}$ ), have been studied and shown to be promising biomarkers for epileptogenesis markers over the last decade. In the KA-induced SE model, Bragin et al. reported the appearance of HFOs in the ipsilateral hippocampi dentate nuclei in rats that later developed epilepsy. The author also described that the appearance of the HFO timing was found to be associated with the delay in the onset of the first seizure (43). The same group reported different types of HFOs named repetitive HFO and spikes (rHFOSs), in which rhythmic spikes at the frequency of $10-16 \mathrm{~Hz}$ with superimposed pathological HFOs $(80-300 \mathrm{~Hz})$. The appearance of rHFOSs in the injured cortex and around the adjacent injured cortex within 2 weeks from the initial insult in the LFPI model rats was reported to more likely develop spontaneous seizures later in life $(20,44)$. Although HFOs are typically detected with invasive intracranial EEG, the advancements in scalp EEG monitoring equipment enable one to record HFOs on scalp EEG in human studies (53). In a cohort of children after a first unprovoked seizure, the presence of scalp ripples can predict the development of epilepsy (45). Further studies, including patients with TBI and febrile seizures, can enhance our knowledge of the role of scalp HFOs as biomarkers of epileptogenesis.

\section{Sleep Spindles and Theta Activity}

Changes in the duration and frequency of the sleep spindles, one of the non-rapid eye movement stage II EEG features, have also been reported as a possible biomarker for epileptogenesis. In the LPFI model, Andrade et al. showed that shortening of the sleep spindles' duration and reducing of their frequency during slow-wave to rapid eye movement sleep transition can predict the development of epilepsy. Furthermore, receiver operating characteristics (ROC) analysis showed that spindle duration of $<2.13$ s ( $86 \%$ sensitivity, $80 \%$ specificity) and frequency of spindle $<9.19 \mathrm{~Hz}$ (64\% sensitivity, $60 \%$ specificity) could be used as biomarkers in differentiating rats with seizures from those without (46). These findings suggest that sleep spindles changes may be the indicators of widespread functional disturbance in the thalamocortical circuits following initial brain insult and could be used as a potential early biomarker for epileptogenesis.

Milkovsky et al. investigated the role of changes in the hippocampal dynamic in five animal models of epileptogenesis 
TABLE 2 | Overview of EEG biomarkers of epileptogenesis.

\begin{tabular}{|c|c|c|c|}
\hline EEG biomarkers & Animal model & Human epilepsy & References \\
\hline $\mathrm{HFO}$ & KA-induced SE model & None & $(43)$ \\
\hline rHFOSs & LFPI model & None & $(20,44)$ \\
\hline Scalp ripples & None & Children after a first unprovoked seizure & $(45)$ \\
\hline Sleep spindles & LPFI model & None & $(46)$ \\
\hline Theta band & Multiple rat and mouse models & None & $(47)$ \\
\hline Epileptiform activity & None & PTE and TSC & $(48-51)$ \\
\hline Background asymmetry & None & Epilepsy after stroke & $(52)$ \\
\hline
\end{tabular}

HFO, High-frequency oscillations; rHFOSs, repetitive HFO and spikes; KA, kainic acid; SE, status epilepticus; LPFI, lateral fluid percussion injury; PTE, posttraumatic epilepsy; TSC, tuberous sclerosis complex.

using the intrahippocampal recording. Among five frequency bands, changes in the dynamic of the theta band on days 2-4 post brain injury showed more than $90 \%$ sensitivity and specificity in predicting the animal which would develop epilepsy (47). Overall this finding is intriguing, but given the invasiveness nature of intracranial electrodes, further studies using noninvasive source modeling may help further validating the finding as early epileptogenesis in the human.

\section{Epileptiform Abnormalities and Background Asymmetry}

The relation between epileptogenesis and scalp EEG findings such as interictal epileptiform discharges, lateralized periodic discharges, background EEG asymmetry, and electrographic seizures has been reported in humans. Although earlier studies in the 20th century do not give much yield in predicting the occurrence of epilepsy in patients with TBI (54), a recent retrospective study reported that during the acute traumatic phase, the presence of EEG interictal and ictal epileptiform abnormalities, such as sporadic interictal epileptiform discharges, lateralized or generalized periodic discharges, and seizures, are correlated with the development of the PTE at 1 year follow up (48). Punia et al. reported a similar result in adult patients that electrographic seizures or lateralized periodic discharges were related with the development of epilepsy after TBI (49). Two prospective multicenter studies investigated the role of scalp EEG in the infants with tuberous sclerosis complex. Among several EEG abnormalities, the author reported that the epileptiform discharges, not the hypsarrhythmia, showed the high positive predictive value and low negative predictive value in the development of epilepsy $(50,51)$. Furthermore, in addition to the interictal epileptiform activities, the presence of background asymmetry plays a role in the early development of epilepsy after stroke (52).

\section{CONCLUSIONS AND FUTURE PERSPECTIVES}

Here we aimed to provide a comprehensive review of recent innovations in the role of neuroimaging and EEG as biomarkers of epileptogenesis after the epileptogenic insult. Identifying biomarkers of epileptogenesis would greatly facilitate not only diagnosis and treatment but also the early prevention of epilepsy in individuals at risk. Although several studies have identified potentially promising biomarkers for early epileptogenesis, such as changes in the amygdala T2 relaxation time, PVSs, TSPOPET, global and local network connectivity reorganization, and HFOs, numerous challenges remain to implement the potential neuroimaging biomarkers to the bedside clinical setting. Firstly, the resolution capacity of human neuroimage is significantly lower than animal neuroimage. Thus, the same biomarker which is reported from animal studies may not be directly replicated in human studies. Secondly, each potential biomarker has both disadvantages and advantages, and it is unrealistic to expect that a single biomarker will epitomize the various types of epileptogenesis. Therefore, a combination of EEG and neuroimaging biomarkers might enhance the predictive power of epileptogenicity. Thirdly, the majority of published data are at the animal stage (summarized in Tables 1, 2). Before all these biomarkers can be utilized in the clinical setting, multicenter studies with standardized acquisition parameters and analysis procedures are needed to validate the robustness of biomarkers.

Currently, ongoing multicenter research studies are aiming to find biomarkers and treatments to prevent epileptogenesis. The European Union 7th Framework-funded project Targets and Biomarkers for Antiepileptogenesis (EPITARGET) is a consortium of 18 partners in nine European countries. In addition, the Epilepsy Bioinformatics Study for Antiepileptogenic Therapy (EpiBios4Rx), National Institute of Neurological Disorders and Stroke funded Centers without Walls study, is a collaborative multicenter international study conducted in the United States, Europe, and Australia. To data, biomarkers for epileptogenesis are still in the initial phase of the process. However, there will be more conclusive innovative EEG and neuroimaging early epileptogenic biomarkers and treatments from the multicenter trials to combat epilepsy in the foreseeable future.

\section{AUTHOR CONTRIBUTIONS}

GC: contributed to the conception and drafting the manuscript, ZZ: drafting the manuscript. $\mathrm{MW}$ and $\mathrm{YG}$ : revising the manuscript. BJ: contributed to the conception, drafting the manuscript, and final approval of the version to be published. TA: drafting the manuscript and revising the manuscript. All authors contributed to the article and approved the submitted version. 


\section{FUNDING}

This study was supported by the National Natural Science Foundation of China (grant number: 82001366),

\section{REFERENCES}

1. Thijs RD, Surges R, O’Brien TJ, Sander JW. Epilepsy in adults. Lancet. (2019) 393:689-701. doi: 10.1016/S0140-6736(18)32596-0

2. Zack MM, Kobau R. National and State estimates of the numbers of adults and children with active epilepsy - United States, 2015. MMWR Morb Mortal Wkly Rep. (2017) 66:821-5. doi: 10.15585/mmwr.mm6631al

3. Chen Z, Brodie MJ, Liew D, Kwan P. Treatment outcomes in patients with newly diagnosed epilepsy treated with established and new antiepileptic drugs: a 30-year longitudinal cohort study. JAMA Neurol. (2018) 75:27986. doi: 10.1001/jamaneurol.2017.3949

4. Löscher W, Schmidt D. Modern antiepileptic drug development has failed to deliver: ways out of the current dilemma. Epilepsia. (2011) 52:65778. doi: 10.1111/j.1528-1167.2011.03024.x

5. Crepeau AZ, Sirven JI. Management of adult onset seizures. Mayo Clin Proc. (2017) 92:306-18. doi: 10.1016/j.mayocp.2016.11.013

6. Pitkänen A, Engel J Jr. Past and present definitions of epileptogenesis and its biomarkers. Neurotherapeutics. (2014) 11:231-41. doi: 10.1007/s13311-014-0257-2

7. Engel J. Biomarkers in epilepsy: foreword. Biomark Med. (2011) 5:52930. doi: $10.2217 / \mathrm{bmm} .11 .63$

8. Engel J Jr, Pitkänen A, Loeb JA, Dudek FE, Bertram EH III, Cole AJ, et al. Epilepsy biomarkers. Epilepsia. (2013) 54(Suppl. 4):61-9. doi: 10.1111/epi.12299

9. Mayeux R. Biomarkers: potential uses and limitations. NeuroRx. (2004) 1:1828. doi: 10.1602/neurorx.1.2.182

10. Shultz SR, Cardamone L, Liu YR, Hogan RE, Maccotta L, Wright DK, et al. Can structural or functional changes following traumatic brain injury in the rat predict epileptic outcome? Epilepsia. (2013) 54:124050. doi: 10.1111/epi.12223

11. Immonen R, Kharatishvili I, Gröhn O, Pitkänen A. MRI biomarkers for post-traumatic epileptogenesis. J Neurotrauma. (2013) 30:1305-9. doi: 10.1089/neu.2012.2815

12. Lutkenhoff ES, Shrestha V, Ruiz Tejeda J, Real C, McArthur DL, Duncan D, et al. Early brain biomarkers of post-traumatic seizures: initial report of the multicentre epilepsy bioinformatics study for antiepileptogenic therapy (EpiBioS4Rx) prospective study. J Neurol Neurosurg Psychiatry. (2020) 91:1154-7. doi: 10.1136/jnnp-2020-322780

13. Duncan D, Barisano G, Cabeen R, Sepehrband F, Garner R, Braimah A, et al. Analytic tools for post-traumatic epileptogenesis biomarker search in multimodal dataset of an animal model and human patients. Front Neuroinform. (2018) 12:86. doi: 10.3389/fninf.2018.00086

14. Feldman RE, Rutland JW, Fields MC, Marcuse LV, Pawha PS, Delman BN, et al. Quantification of perivascular spaces at 7T: a potential MRI biomarker for epilepsy. Seizure. (2018) 54:11-8. doi: 10.1016/j.seizure.2017.11.004

15. Choy M, Dubé CM, Patterson K, Barnes SR, Maras P, Blood AB, et al. A novel, noninvasive, predictive epilepsy biomarker with clinical potential. J Neurosci. (2014) 34:8672-84. doi: 10.1523/jneurosci.4806-13.2014

16. Curran MM, Haddad E, Patterson KP, Choy M, Dubé CM, Baram TZ, et al. Epilepsy-predictive magnetic resonance imaging changes following experimental febrile status epilepticus: are they translatable to the clinic? Epilepsia. (2018) 59:2005-18. doi: 10.1111/epi.14561

17. Huang YC, Weng HH, Tsai YT, Huang YC, Hsiao MC, Wu CY, et al. Periictal magnetic resonance imaging in status epilepticus. Epilepsy Res. (2009) 86:72-81. doi: 10.1016/j.eplepsyres.2009.05.011

18. Sierra A, Laitinen T, Gröhn O, Pitkänen A. Diffusion tensor imaging of hippocampal network plasticity. Brain Struct Funct. (2015) 220:781801. doi: 10.1007/s00429-013-0683-7

19. Wang H, Huang Y, Coman D, Munbodh R, Dhaher R, Zaveri HP, et al. Network evolution in mesial temporal lobe epilepsy revealed by the Natural Science Foundation of Zhejiang Province (grant number: LQ20H090019), and China and Zhejiang Medical Science and Technology Project (grant number: 2021KY020), China. diffusion tensor imaging. Epilepsia. (2017) 58:824-34. doi: 10.1111/epi. 13731

20. Reid AY, Bragin A, Giza CC, Staba RJ, Engel J Jr. The progression of electrophysiologic abnormalities during epileptogenesis after experimental traumatic brain injury. Epilepsia. (2016) 57:1558-67. doi: 10.1111/epi.13486

21. Li L, He L, Harris N, Zhou Y, Engel J, Jr., Bragin A. Topographical reorganization of brain functional connectivity during an early period of epileptogenesis. Epilepsia. (2021) 62:1231-43. doi: 10.1111/epi.16863

22. Christiaen E, Goossens MG, Raedt R, Descamps B, Larsen LE, Craey $\mathrm{E}$, et al. Alterations in the functional brain network in a rat model of epileptogenesis: a longitudinal resting state fMRI study. Neuroimage. (2019) 202:116144. doi: 10.1016/j.neuroimage.2019.116144

23. Bertoglio D, Jonckers E, Ali I, Verhoye M, Van der Linden A, Dedeurwaerdere $\mathrm{S}$. In vivo measurement of brain network connectivity reflects progression and intrinsic disease severity in a model of temporal lobe epilepsy. Neurobiol Dis. (2019) 127:45-52. doi: 10.1016/j.nbd.2019.02.012

24. Lee EM, Park GY, Im KC, Kim ST, Woo CW, Chung JH, et al. Changes in glucose metabolism and metabolites during the epileptogenic process in the lithium-pilocarpine model of epilepsy. Epilepsia. (2012) 53:8609. doi: $10.1111 / \mathrm{j} .1528-1167.2012 .03432 . \mathrm{x}$

25. Filibian M, Frasca A, Maggioni D, Micotti E, Vezzani A, Ravizza T. In vivo imaging of glia activation using $1 \mathrm{H}$-magnetic resonance spectroscopy to detect putative biomarkers of tissue epileptogenicity. Epilepsia. (2012) 53:1907-16. doi: 10.1111/j.1528-1167.2012.03685.x

26. van der Hel WS, van Eijsden P, Bos IW, de Graaf RA, Behar KL, van Nieuwenhuizen O, et al. In vivo MRS and histochemistry of status epilepticusinduced hippocampal pathology in a juvenile model of temporal lobe epilepsy. NMR Biomed. (2013) 26:132-40. doi: 10.1002/nbm.2828

27. Pascente R, Frigerio F, Rizzi M, Porcu L, Boido M, Davids J, et al. Cognitive deficits and brain myo-Inositol are early biomarkers of epileptogenesis in a rat model of epilepsy. Neurobiol Dis. (2016) 93:146-55. doi: 10.1016/j.nbd.2016.05.001

28. Croall I, Smith FE, Blamire AM. Magnetic resonance spectroscopy for traumatic brain injury. Top Magn Reson Imaging. (2015) 24:26774. doi: 10.1097/rmr.0000000000000063

29. Immonen $\mathrm{R}$, Harris $\mathrm{NG}$, Wright $\mathrm{D}$, Johnston $\mathrm{L}$, Manninen $\mathrm{E}$, Smith G, et al. Imaging biomarkers of epileptogenecity after traumatic brain injury - Preclinical frontiers. Neurobiol Dis. (2019) 123:75-85. doi: 10.1016/j.nbd.2018.10.008

30. Goffin K, Van Paesschen W, Dupont P, Van Laere K. Longitudinal microPET imaging of brain glucose metabolism in rat lithium-pilocarpine model of epilepsy. Exp Neurol. (2009) 217:205-9. doi: 10.1016/j.expneurol.2009.02.008

31. Guo Y, Gao F, Wang S, Ding Y, Zhang $\mathrm{H}$, Wang J, et al. In vivo mapping of temporospatial changes in glucose utilization in rat brain during epileptogenesis: an 18F-fluorodeoxyglucose-small animal positron emission tomography study. Neuroscience. (2009) 162:972-9. doi: 10.1016/j.neuroscience.2009.05.041

32. Jupp B, Williams J, Binns D, Hicks RJ, Cardamone L, Jones $\mathrm{N}$, et al. Hypometabolism precedes limbic atrophy and spontaneous recurrent seizures in a rat model of TLE. Epilepsia. (2012) 53:1233-44. doi: 10.1111/j.1528-1167.2012.03525.x

33. Syvänen $S$, Labots $M$, Tagawa $Y$, Eriksson J, Windhorst $A D$, Lammertsma AA, et al. Altered GABAA receptor density and unaltered blood-brain barrier transport in a kainate model of epilepsy: an in vivo study using 11C-flumazenil and PET. J Nucl Med. (2012) 53:1974-83. doi: 10.2967/jnumed.112.104588

34. Vivash L, Gregoire MC, Bouilleret V, Berard A, Wimberley C, Binns $\mathrm{D}$, et al. In vivo measurement of hippocampal GABAA/cBZR density with [18F]-flumazenil PET for the study of disease progression in an animal model of temporal lobe epilepsy. PLOS ONE. (2014) 9:e86722. doi: 10.1371/journal.pone.0086722 
35. Morimoto $\mathrm{K}$, Watanabe $\mathrm{T}$, Ninomiya $\mathrm{T}$, Hirao $\mathrm{T}$, Tanaka A, Onishi $\mathrm{T}$, et al. Quantitative evaluation of central-type benzodiazepine receptors with [(125)I]Iomazenil in experimental epileptogenesis: II. The rat cortical dysplasia model. Epilepsy Res. (2004) 61:113-8. doi: 10.1016/j.eplepsyres.2004.07.006

36. Bertoglio D, Verhaeghe J, Santermans E, Amhaoul H, Jonckers E, Wyffels L, et al. Non-invasive PET imaging of brain inflammation at disease onset predicts spontaneous recurrent seizures and reflects comorbidities. Brain Behav Immun. (2017) 61:69-79. doi: 10.1016/j.bbi.2016.12.015

37. Bertoglio D, Amhaoul H, Goossens J, Ali I, Jonckers E, Bijnens T, et al. TSPO PET upregulation predicts epileptic phenotype at disease onset independently from chronic TSPO expression in a rat model of temporal lobe epilepsy. Neuroimage Clin. (2021) 31:102701. doi: 10.1016/j.nicl.2021.102701

38. Dubé CM, Brewster AL, Richichi C, Zha Q, Baram TZ. Fever, febrile seizures and epilepsy. Trends Neurosci. (2007) 30:4906. doi: 10.1016/j.tins.2007.07.006

39. Yokoi S, Kidokoro $H$, Yamamoto $H$, Ohno A, Nakata $T$, Kubota $T$, et al. Hippocampal diffusion abnormality after febrile status epilepticus is related to subsequent epilepsy. Epilepsia. (2019) 60:1306-16. doi: 10.1111/epi. 16059

40. Duncan JS. Imaging the brain's highways-diffusion tensor imaging in epilepsy. Epilepsy Curr. (2008) 8:85-9. doi: 10.1111/j.1535-7511.2008.00249.x

41. Ogawa S, Lee TM, Kay AR, Tank DW. Brain magnetic resonance imaging with contrast dependent on blood oxygenation. Proc Natl Acad Sci USA. (1990) 87:9868-72. doi: 10.1073/pnas.87.24.9868

42. Vezzani A, French J, Bartfai T, Baram TZ. The role of inflammation in epilepsy. Nat Rev Neurol. (2011) 7:31-40. doi: 10.1038/nrneurol.2010.178

43. Bragin A, Wilson CL, Almajano J, Mody I, Engel J, Jr. High-frequency oscillations after status epilepticus: epileptogenesis and seizure genesis. Epilepsia. (2004) 45:1017-23. doi: 10.1111/j.0013-9580.2004.17004.x

44. Bragin A, Li L, Almajano J, Alvarado-Rojas C, Reid AY, Staba RJ, et al. Pathologic electrographic changes after experimental traumatic brain injury. Epilepsia. (2016) 57:735-45. doi: 10.1111/epi. 13359

45. Klotz KA, Sag Y, Schonberger J, Jacobs J. Scalp ripples can predict development of epilepsy after first unprovoked seizure in childhood. Ann Neurol. (2021) 89:134-42. doi: 10.1002/ana. 25939

46. Andrade $\mathrm{P}$, Nissinen J, Pitkänen A. Generalized seizures after experimental traumatic brain injury occur at the transition from slow-wave to rapid eye movement sleep. J Neurotrauma. (2017) 34:1482-7. doi: 10.1089/neu.2016.4675

47. Milikovsky DZ, Weissberg I, Kamintsky L, Lippmann K, Schefenbauer $\mathrm{O}$, Frigerio $\mathrm{F}$, et al. Electrocorticographic dynamics as a novel biomarker in five models of epileptogenesis. J Neurosci. (2017) 37:4450-61. doi: 10.1523/jneurosci.2446-16.2017

48. Kim JA, Boyle EJ, Wu AC, Cole AJ, Staley KJ, Zafar S, et al. Epileptiform activity in traumatic brain injury predicts post-traumatic epilepsy. Ann Neurol. (2018) 83:858-62. doi: 10.1002/ana.25211

49. Punia V, Fitzgerald Z, Zhang X, Huynh H, Bena J, Morrison S, et al. Electroencephalographic biomarkers of epilepsy development in patients with acute brain injury: a matched, parallel cohort study. Ann Clin Transl Neurol. (2019) 6:2230-9. doi: 10.1002/acn3.50925

50. Wu JY, Peters JM, Goyal M, Krueger D, Sahin M, Northrup H, et al. Clinical electroencephalographic biomarker for impending epilepsy in asymptomatic tuberous sclerosis complex infants. Pediatr Neurol. (2016) 54:29-34. doi: 10.1016/j.pediatrneurol.2015. 09.013

51. Wu JY, Goyal M, Peters JM, Krueger D, Sahin M, Northrup H, et al. Scalp EEG spikes predict impending epilepsy in TSC infants: a longitudinal observational study. Epilepsia. (2019) 60:2428-36. doi: 10.1111/epi.16379

52. Bentes C, Martins H, Peralta AR, Morgado C, Casimiro C, Franco AC, et al. Early EEG predicts poststroke epilepsy. Epilepsia Open. (2018) 3:20312. doi: 10.1002/epi4.12103

53. Fan Y, Dong L, Liu X, Wang H, Liu Y. Recent advances in the noninvasive detection of high-frequency oscillations in the human brain. Rev Neurosci. (2021) 32:305-21. doi: 10.1515/revneuro-2020-0073

54. Jennett B, Van De Sande J. EEG prediction of post-traumatic epilepsy. Epilepsia. (1975) 16:251-6. doi: 10.1111/j.1528-1157.1975.tb06055.x

Conflict of Interest: The authors declare that the research was conducted in the absence of any commercial or financial relationships that could be construed as a potential conflict of interest.

Publisher's Note: All claims expressed in this article are solely those of the authors and do not necessarily represent those of their affiliated organizations, or those of the publisher, the editors and the reviewers. Any product that may be evaluated in this article, or claim that may be made by its manufacturer, is not guaranteed or endorsed by the publisher.

Copyright (c) 2021 Chen, Zhang, Wang, Geng, Jin and Aung. This is an open-access article distributed under the terms of the Creative Commons Attribution License (CC $B Y)$. The use, distribution or reproduction in other forums is permitted, provided the original author(s) and the copyright owner(s) are credited and that the original publication in this journal is cited, in accordance with accepted academic practice. No use, distribution or reproduction is permitted which does not comply with these terms. 